A N N A L E S Annales de Bretagne et des Pays de l'Ouest

Anjou. Maine. Poitou-Charente. Touraine

112-1 | 2005

Varia

\title{
Prisons et prisonniers en Anjou au bas Moyen Âge
}

Isabelle Mathieu

\section{OpenEdition}

Journals

Édition électronique

URL : http://journals.openedition.org/abpo/1146

DOI : $10.4000 /$ abpo.1146

ISBN : 978-2-7535-1497-3

ISSN : 2108-6443

Éditeur

Presses universitaires de Rennes

Édition imprimée

Date de publication : 20 mars 2005

Pagination : 147-169

ISBN : 978-2-7535-0125-6

ISSN : 0399-0826

Référence électronique

Isabelle Mathieu, «Prisons et prisonniers en Anjou au bas Moyen Âge », Annales de Bretagne et des Pays de l'Ouest [En ligne], 112-1 | 2005, mis en ligne le 20 mars 2007, consulté le 19 avril 2019. URL http://journals.openedition.org/abpo/1146 ; DOI : 10.4000/abpo.1146 


\title{
Prisons et prisonniers en Anjou au bas Moyen Âge
}

\author{
Isabelle MATHIEU \\ Étudiante, université d'Angers
}

Si les ouvrages concernant le monde et la population carcérales sont nombreux pour l'époque moderne et contemporaine ${ }^{1}$, on ne peut en revanche que constater, à quelques exceptions près, les lacunes de l'historiographie pour le Moyen $\widehat{A} \mathrm{e}^{2}$. En effet, la bibliographie relative à un tel sujet nous présente des ouvrages qui sont aujourd'hui un peu anciens et qui mériteraient d'être renouvelés au vu de nouvelles pistes de recherche. Il est aisé de comprendre l'appréhension des chercheurs quant à ce genre d'études. Sujet tiraillé entre de multiples disciplines telles l'histoire, l'his-

1. CARBASSE, Jean-Marie, Introduction historique au droit pénal, Paris, 1990; PETIT, JaquesGuy (dir.), Histoire des galères, bagnes et prisons en France de l'Ancien Régime, Paris, 2002; GARNOT, Benoît, Vivre en prison au XVIII siècle, Paris, 1994; DEYON, Pierre, Le Temps des prisons, Université de Lille III, éditions universitaires, Lille, 1975; FoucAuLt, Michel, Surveiller et punir, Paris, 1975; PETIT, Jaques-Guy, L'amendement ou l'entreprise de reforme morale des prisonniers en France au XIX $x^{e}$ siècle, Paris, 1982; PETIT, Jaques-Guy, Histoire des prisons en France (1789-2000), Paris, 2002.

2. Gauvard, Claude, Crime, état et société en France à la fin du Moyen Âge, 2 tomes, Paris, 1991; GonTHIER, Nicole, "Prisons et prisonniers à Lyon aux XIV et XV siècles ", Mémoires de la société pour l'histoire du droit et des institutions des anciens pays bourguignons, comtois et romands, fascicule 39, 1982, p. 15-30; GONTHIER, Nicole, " La répression et le crime à la fin du Moyen Âge ", Mémoires de la société pour l'histoire du droit et des institutions des anciens pays bourguignons, comtois et romands, fascicule 47, 1990, p. 115130 ; GonTHIER, Nicole, "La violence judiciaire à Dijon à la fin du Moyen Âge ", Mémoires de la société pour l'histoire du droit et des institutions des anciens pays bourguignons, comtois et romands, fascicule 50, 1993, p. 19-34; GRAND, Roger, " La prison et la notion d'emprisonnement dans l'ancien droit ", Nouvelle revue historique de droit français et étranger, 1940, p. 58-87; PORTEAU-BITKER, Annik, "L'emprisonnement dans le droit laïque du Moyen Âge ", Nouvelle revue historique de droit français et étranger, 1968, p. 211-245; PORTEAUBITKER, Annik, "Criminalité et délinquance féminines dans le doit pénal des XIII et XIV siècles ", Revue historique de droit français et étranger, 1980, p. 13-56; OTIs-CouR, Leah, "L'exemplarité de la peine en question, la pratique de la peine cachée dans le midi de la France au Xve siècle ", Revue historique de droit français et étranger, 2002, p. 179-186; ToureILle, Valérie, "Prison ", Dictionnaire du Moyen Âge, GAUvard, Claude, LiBERA, Alain DE et ZinK, Michel (dir.), Paris, 2002, p. 1149; VINCENT-CASSY, Mireille, " Prison et châtiments à la fin du Moyen Âge ", Les Marginaux et les exclus dans l'histoire, Cahier Jussieu, Université Paris VII, Paris, 1979, p. 262-274. 
toire du droit, la sociologie, l'anthropologie, il s'agit aussi pour le chercheur de se confronter aux difficultés paléographiques et à l'ésotérisme du vocabulaire propre aux sources judiciaires du bas Moyen Âge.

C'est au cours du dépouillement systématique de registres d'assises ${ }^{3}$ que nous nous sommes rendus compte de la possibilité de collecter des informations relatives au monde de la prison et des prisonniers en Anjou à la fin du Moyen Âge, et de ce fait d'offrir l'esquisse d'une synthèse sur un tel sujet. Par ailleurs, la consultation de la coutume d'Anjou et du Maine nous a permis d'enrichir nos recherches d'un point de vue purement législatif et juridique. Dépouillé à $80 \%$, notre corpus documentaire est composé d'un total de 71 cotes $^{4}$ et permet d'appréhender entre 45 et 50 lieux géographiques différents ${ }^{5}$ répartis sur le territoire angevin avec une concentration dans un rayon d'une quarantaine de kilomètres autour d'Angers.

Les registres ${ }^{6}$ d'assises sont des documents dans lesquels étaient annotés tous les délits ainsi que les délibérations des affaires qui passaient devant les tribunaux seigneuriaux. Les sources nous montrent que ces séances d'assises étaient l'occasion d'affrontements entre les seigneurs et leurs sujets mais aussi entre les sujets eux-mêmes. Réclamations d'aveux, de foi et hommages, devoirs et cens non payés, dettes, pâtures illicites, chemins obstrués, délits de chasse et de pêche, dégradations diverses, injures, coups et blessures... sont quelques exemples de litiges quotidiens auxquels devaient faire face le personnel des justices seigneuriales.

Une simple lecture de ces registres permet d'emblée de constater que l'usage de la prison n'est pas systématique et que l'enfermement d'un individu répond à des règles strictement définies. Bien évidemment, les cas d'emprisonnement abusif ne sont pas à exclure mais en ce qui nous concerne les sources ne nous en ont livré aucun cas.

Il est clair que l'on est loin de l'image d'Épinal du cachot dans lequel un méchant seigneur enferme un ennemi dans l'obscurité et l'humidité ${ }^{7}$. L'étude se situe dans le cadre de prisons seigneuriales ${ }^{8}$. Le sens premier du terme

3. Ces dépouillements sont effectués dans le cadre d'une thèse concernant les justices seigneuriales en Anjou et dans le Maine au bas Moyen Âge (milieu XIV ${ }^{\mathrm{e}}$-milieu $\mathrm{XVI}^{\mathrm{e}}$ siècle).

4. Toutes ces cotes, réparties surtout dans les séries G, H, E, J, sont conservées aux Archives départementales de Maine-et-Loire.

5. Il serait pour le moment prématuré de parler de 45 à 50 seigneuries différentes car toutes les vérifications n'ont pas encore été faites à ce sujet. Par ailleurs, nous avons parfois plusieurs cotes qui concernent le même lieu ce qui justifie l'inadéquation entre d'une part le nombre de cotes et de l'autre le nombre de lieux concernés par notre étude.

6. Notre corpus renferme aussi quelques registres d'amendes.

7. Ces quelques mots qui résument très bien l'opinion souvent véhiculée à propos de la prison médiévale sont tirés de l'article de VINCENT-CASSY, Mireille, "Prison et châtiments à la fin du Moyen Âge ", Les Marginaux et les exclus..., op. cit., p. 262.

8. En effet, Annik ProteAu-BITKER note que celles-ci peuvent être royales, municipales, ecclésiastiques mais que face à un pouvoir unique et centralisé, la multitude des prisons importe peu et facilite plutôt l'exercice d'une bonne justice, "L'emprisonnement dans le droit laïque du Moyen Âge ", Nouvelle revue..., op. cit., p. 212. 
prison ou chartre ${ }^{9}$ est bien de définir globalement un lieu $\operatorname{clos}^{10}$. Il est aussi courant d'associer au mot prison celui de peine. C'est ici qu'intervient une différence notable quant à l'acception de ce terme entre d'une part le droit canonique ${ }^{11}$ et d'autre part le droit laïque. L'Église, étant privée des peines corporelles et de la peine de mort ${ }^{12}$, trouve dans la prison le moyen par excellence de punir les récalcitrants ${ }^{13}$. En revanche, pour le droit et les tribunaux séculiers, fidèles au droit romain, la prison est avant tout considérée comme une possibilité offerte au personnel judiciaire de protéger la société en écartant les individus jugés dangereux le temps qu'un jugement définitif soit rendu. L'enfermement des individus intervient aussi lorsque ces derniers ne peuvent pas fournir de cautions ${ }^{14}$ morales à leur élargissement ou que l'affaire dans laquelle ils sont impliqués est jugée trop grave ${ }^{15}$.

9. Ce terme est un synonyme de prison. Nous l'avons rencontré dans le registre coté 1 Hs B 176 au f 122 "Collecte femme de feu Emery Caunet nagueres demourant Notre Dame d'Angers prisonniere en la chartre et prison de notre aumosnerie acusée d'avoir prins et emblé à une femme appellée Jehanne Coingneue... " (1391).

10. Une nuance doit être apportée. Annik Proteau-Bitker souligne que cet état de privation de liberté présente la particularité de comporter plusieurs degrés. Au Moyen Âge, le fait d'emprisonner quelqu'un n'implique pas forcément l'idée de clôture étroite qui nous est familière aujourd'hui : l'emprisonnement en " prison fermée " est une incarcération véritable tandis que la " prison ouverte " n'est pas une incarcération mais une mise en arrêt, "L'emprisonnement dans le droit laïque du Moyen Âge ", Nouvelle revue..., op. cit., p. 214-215. En ce qui nous concerne, nous avons pris le parti de ne nous intéresser qu'aux " véritables détentions " en prison entre quatre murs. Ces termes de " prison ouverte, prison fermée " n'apparaissent pas dans nos sources. Néanmoins, l'idée avancée de détention pure et de mise en arrêt transparaît lorsque le personnel judiciaire mentionne une " prinse au corps " suivie d'une injonction à comparaître ultérieurement.

11. Roger GRAND dans " La prison et la notion d'emprisonnement dans l'ancien droit ", Nouvelle revue..., op. cit., résume (p. 63) la théorie canonique. Pour lui la prison est une peine; c'est même la plus grave que puissent prononcer les tribunaux ecclésiastiques, et elle porte le cachet de toutes les peines prononcées par l'Église : elle a une vertu " médicinale "; elle vise à corriger autant qu'à punir le coupable.

12. En fait, elle peut les prononcer, mais en délègue l'exécution au "bras séculier ".

13. Jean-Marie CARBASSE dans son ouvrage intitulé Introduction historique..., op. cit., note (p. 208) que la prison devait permettre au condamné en situation de méditer sur ses fautes, d'en éprouver le repentir, de s'ouvrir à la grâce divine; " le pain de tristesse et l'eau d'angoisse " qui constituaient l'ordinaire du prisonnier, devaient lui permettre de se purifier de ses fautes et ainsi de sauver son âme.

14. La coutume nous en expose un exemple dans le cadre d'un aveu, BEAUTEMPS-BEAUPRE, Charles-Jean, Coutumes et institutions de l'Anjou et du Maine antérieures au XVI siècle, coutumes et styles, première partie, 4 tomes, 1877 en particulier au tome 4 , chapitre $\mathrm{M}$, titre XXX " des causes d'adveu et contradveu ", paragraphe 202, p. 462 " et pour ce que ledit adveu denote crime et accusation de larrecin, la chose advouée est arrestée et sequestrée, et n'en doit estre faicte delivrance à l'une ne à l'autre des parties jusquez en deffinitive : et doit l'advoueur bailler plege en son adveu, ou en deffault de ce faire estre constitué prinsonnier jusquez à ce qu'il ayt baillé caution suffisante de fournir et obeyr à droit. Et semblablement est tenu celuy sur lequel la chose aura esté advouée bailler plege et caution, ou sera fait prinsonnier. Toutesfois s'il n'est suspect et que bonnement il ne puisse lors fournir de plege sequestrant la chose advouée, on pourra prendre caution juratoyre du deffendeur et contradvoueur de soy rendre sub pena convicti, avec obligation et promesse de fournir et obeyr à droit : et doit eslire domicille ".

15. La jurisprudence, par les confessions faites avant l'exécution des peines corporelles 
Ainsi, l'incarcération dure le temps de l'instruction et permet tout simplement au personnel des justices seigneuriales d'être certain que les prévenus ne leur échapperont pas ${ }^{16}$. L'emprisonnement ne représente donc bien qu'une étape dans le long cheminement qui mène à la peine définitive. On enferme avant de pendre, de brûler, de bannir, de mutiler, d'exposer au pilori ou bien de relâcher. De même, il existe une relation très étroite entre la prison et l'amende. En effet, il est possible, dans le cas de délits mineurs, de convertir une détention en prison en une simple amende pécuniaire. L'idée que la prison ne constitue pas une peine ${ }^{17}$ a perduré jusqu'à la fin de l'Ancien Régime ${ }^{18}$. La coutume d'Anjou illustre parfaitement cet état de fait.

\section{Une approche juridique du sujet : prisons et prisonniers à travers la coutume}

Aucun chapitre n'est consacré en propre à cette notion d'emprisonnement vécu comme une peine et, c'est éparpillées au gré de quelques passages que l'on peut trouver des références à la prison et aux prisonniers. Par ailleurs, la coutume prévoit clairement tout une gamme de peines en fonction des délits et la prison en est visiblement absente. A contrario, sont mentionnées les peines corporelles ${ }^{19}$ et les peines pécuniaires ${ }^{20}$.

par les prisonniers incarcérés sur les motifs de leur incarcération, confirme et complète encore le contenu des chartes et coutumiers sur la nature exacte des crimes et des délits qui conduisent leurs auteurs en "prison fermée " : l'empoisonnement, le vol, même quand il n'est pas accompagné de blessures, les exactions et abus de justice, la sodomie, le crime de faux, la sorcellerie, la complicité dans un meurtre, le proxénétisme, le rapt, le meurtre. PORTEAU-BITKER, Annik, «L'emprisonnement dans le droit laïque du Moyen Âge », Nouvelle revue..., op. cit., p. 222.

16. Nicole GoNTHIER dans " Prisons et prisonniers à Lyon aux XIv et XV $v^{\mathrm{e}}$ siècles ", Mémoires de la société pour l'histoire..., op. cit., p. 17 note que la prison est le seul moyen qu'ont la police d'abord, la justice ensuite, pour conserver à leur disposition des délinquants.

17. Roger GRAND dans " La prison et la notion d'emprisonnement dans l'ancien droit ", Nouvelle revue..., op. cit., souligne que " le Moyen Âge et les derniers siècles de la monarchie s'en seraient tenus au principe ancien : la prison sert à garder, non à punir ".

18. Jean-Marie CARBASSE pense que le droit canonique s'est donné comme objectif dès le Moyen Âge l'amendement des coupables tandis que le droit laïque a très longtemps considéré la peine comme exclusivement expiatoire et compensatrice, la notion d'amendement ne faisant une timide apparition qu'à la fin de l'Ancien Régime, Introduction historique..., op. cit., p. 206.

19. Voir à ce propos BEAUTEMPS-BAUPRE, Charles-Jean, Coutumes et institutions..., op. cit., tome 1, chapitre F, titre XVIII, p. 502-508 intitulé « De paines corporelles ». Le passage est divisé en 32 paragraphes différents dans chacun desquels, en fonction des délits abordés et de la qualité des délinquants, les châtiments corporels sont clairement explicités. Ainsi, par exemple nous trouvons que " cieulx qui sont convaincuz et actains d'avoir commis crime de leze majesté doivent estre decollez et le corps pendu au gibet ou equartelé " mais aussi « sacrilleges qui desrobent les Églises doivent estre trainez et penduz et ne peuvent joir de la liberté de l'Église " ou bien que pour " de connins emblez en garenne et de poisson en estanc par nuyt, le larron doit estre pendu ".

20. De même se référer à BEAUTEMPS-BAUPRE, Charles-Jean, ibidem, et en particulier toujours au tome 1, chapitre F, titre XIX, p. 508-515 intitulé "De paines pecunielles ". On constate, cette fois-ci, une division en 36 paragraphes différents dans lesquels, toujours 
La lecture des coutumes d'Anjou et du Maine permet néanmoins d'appréhender quelques aspects de la prison et du statut de prisonnier. Sont prises en compte les différences de traitement selon la qualité de l'individu, laïc ou clerc :

« Nul clerc ou ecclesiastique personne ne doit estre prins ou arresté par le juge secullier ou ses officiers, s'il n'est prins au present meffait ou en suyte, ou suy à cry, et estre rendu à l'Église lui premierement requis. Et s'il est ainsi prins et arresté par ladicte justice seculliere et sans avoir tonsure de clerc, et il soit doubte ou il soit clerc ou nom, la preuve s'en fera davant le juge secullier, les gens de l'Église presens ou absens. Et s'il est requis par le juge de l'Église en informant qu'il soit clerc, le juge secullier le lui doibt rendre avecques les cas pour lesquielx il est detenu em prinson ${ }^{21}$."

La détention ${ }^{22}$, le temps de l'instruction d'une affaire, est clairement légitimée, de même que sont mentionnés les rares cas dans lesquels l'emprisonnement est visiblement recommandé ${ }^{23}$. Par ailleurs, la coutume explicite les risques auxquels les détenus s'exposeraient en cas d'évasion ${ }^{24}$. Les textes prévoient également de quelle manière l'interrogatoire d'un prisonnier doit se dérouler :

«Par le greffier ou garde de la chartre si tost que le delinquant est en prison, doit estre registré le jour qu'il est amené; par quel sergent : par infourmacion d'office ou denonciement; et de quoy il est accusé. Et ce fait le juge à tenir son procès doit tenir tel ordre qui s'ensuit : tel jour a esté interrogué par ledit juge tel prisonnier aagé de tel aage sur le cas dessusdit, lequel après son

en fonction des délits et de la qualité des délinquants, des peines pécuniaires sont prévues. En guise d'exemples notons que « une homme coustumier qui bat le provost de son seigneur ou son sergent de son houstel qui porte les clefs il en fait LXs d'amende au seigneur et l'amende à celui a esté feru selon le dommaige à sa prouve " ou bien encore " en injures desloyaux comme appellez autre traitre, laron, meurtrier ou autre injure equipolent à ce, a amende de VI livres ou Maine et LXs en Anjou. Et en simples injures a amende de loy".

21. Ibid., tome 2, chapitre F, titre II " d'evesques, de clercs et religieulx ", paragraphe 22, p. 40. Ce passage fait aussi clairement référence au privilège du for ecclésiastique dont bénéficiaient les clercs. La qualité d'un individu décidait pour lui qu'il soit justiciable de la justice laïque ou de la justice de l'Église.

22. Ibid., tome 1, chapitre E (1411), quatrième partie " la quarte partie de amendes, prouffitz, forfaictures, parties de fief, aventures et confiscations que les seigneurs ont sur leurs hommes et en quel cas etc..." ", paragraphe 83, p. 433 " s'aucun larron prins ou emprisonné pour aucun larroncin confesse son delict et actuse autre celui qu'il actuse n'est pas pour ce atainct ne prouvé du cas ; mais la justice le peut bien prendre, emprisonner et examiner pour savoir la verité du cas".

23. Ibid., tome 4, chapitre M, titre XXVIII « des requestes de lettres, bannies et subhastations ", paragraphe 196 , p. 458 " et est assavoir que quant le decret est adjugé pour une somme d'argent content, on ne baille ne adjuge jamais le decret que celluy à qui il est adjugé ne paye content la somme à ceulx à qui elle appartient, ou qu'il consigne en court, ou qu'il apporte quictance de celluy ou celle à qui elle appartient; autrement il peut estre prinsonnier jusquez à satisfation de la somme à quoy il a mis icelles choses à pris ".

24. Ibid., tome 1, chapitre C (1385), paragraphe 85 " de briser prison ", p. 303 " si aucun estoit en prison pour souspecon de murtre ou de larrecin ou d'autre meffait dont il deust prendre mort et il s'en alast et foist de la prison, tout n'eust il pas fait le meffait, si en seroit il prouvé et ataint come se il avoit fait le meffait". 
serement fait de dire verité, et qu'il a par nous esté adverti de la coustume du pais, a congneu sans gehayne qu'il est natif de tel lieu : filz de tel pere, qui est de tel mestier; qu'il a esté nourri avecques telles gens de telle condicion; et y a servy de telles choses ou temps passé, et sert à present de telles choses, et a intencion ou temps à venir de telles. Et s'il est absent de son pays, soit enquise la cause de son absence. Et au regard desdiz cas, dit qu'il est ainsi et non autrement, et soit enquis s'il en veult croire telz et telz, et la commune renommée, et sur ce escripre sa responce. Et ont esté presens à ce telz et telz. Tel jour ensuivant a esté audit prisonnier sa confession leue, et icelle de mot à mot entendue, a confessé par son serement qu'elle est vraye, et l'avoir confessé en telle fourme sans force ne violence : et oultre a confessé telz autres cas : presens à ce, telz, etc. Tel jour ensuivant par ledit juge ont esté faiz assembler telz advocatz et conseillers, en la presence desquelz les infourmacions et confessions dessusdictes ont esté leues, et à eulx demandé conseil et advis qu'il estoit à faire pour acomplissement de justice : lesquelz ont esté d'oppinion que considerées telles choses, il est expedient pour le bien de justice que ledit tel soit mis en question extraordinaire, affin que par sa bouche on saiche plus avant la verité du cas. En ensuivant laquelle oppinion, ledit prisonnier a esté despouillé. Et pour ce qu'il a declairé estre clerc, et que telz barbiers ont rapporté par leur serement qu'il a couronne faicte o pointe de rasouer, l'avons rendu à l'official et baillé à son promoteur avecques la copie desdictes infourmacion et confession. Ou s'il n'est clerc, a esté mis en gehaine; laquelle il a soufferte et endurée sans autre chose confesser : ou, a confessé telle chose et persevere lui estant hors. Neantmoins lesquelles infourmacions et confessions lesdiz telz ont esté d'oppinion que ledit tel a desservy telle pugnicion, à laquelle recevoir ledit juge l'a condampné ${ }^{25}$."

Ce passage nous montre que l'interrogatoire répondait à une procédure strictement définie et élaborée ${ }^{26}$ et apporte une preuve supplémentaire que le Moyen Âge est loin d'être une époque où ne régnaient que terreur et loi du plus fort.

Par ailleurs, certains droits des prisonniers ainsi que les restrictions dues à leur statut de détenus sont envisagés ${ }^{27}$. Ainsi, un paragraphe est

25. Ibid., tome 4, chapitre L, vingtième partie " des aplegemens et contraplegemens, tans simples, privilegiés, en tiers pié, en declinant, que autres : de restablissemens : de monstrées : de recreance : et de la fourme de proceder esdictes choses ", paragraphe 410 "l'ordre de examiner prisonnier ", p. 311-312.

26. Notre dépouillement nous a permis de trouver quatre cotes renfermant des procès relatés dans le détail où l'on voit bien que cette procédure de l'interrogatoire est suivie : Arch. dép. de Maine-et-Loire, $1^{\mathrm{E}} 1174$, f ${ }^{\circ}$ 42, (1464-1506); G 575, (1501-1511); G 576, (1513); H 83, (1482).

27. BeautemPs-Baupre, Charles-Jean, Coutumes et institutions..., op. cit., tome 2, huitième partie, titre V " des dillatoires nommés de non recevoir ", paragraphe 1142, p. 432 : " homme prins ne doit pas respondre s'il n'est prins par cas dont il puisse avoir sa delivrance o pleges, et puis respondra après ", et paragraphe 1143 , p. 433 : " si aucun est em prinson detenu, il n'est pas tenu de respondre des querelles feodaux jusques à tant qu'il soit de la chartre delivré : excepté touteffoix pour octasion d'icelle chose pour laquelle il seroit emprisonné ou il soit em prinson ou il soit baillé en garde ", de même au tome 4, chapitre L, vingtième partie " des aplegemens et contraplegemens, tant simples, privilegiés, en tiers pié, en declinant, que autres : de restablissemens : de monstrées : de recreance : et de la fourme de proceder esdictes choses ", paragraphe 458, p. 332 : 
consacré à leurs biens ${ }^{28}$, à savoir ce qu'ils adviennent durant le temps de l'incarcération et après la remise en liberté de l'individu dans l'hypothèse où celle-ci intervient. Enfin, les textes réglementent la garde des prisonniers ${ }^{29}$ et les conflits de juridictions qui peuvent survenir entre les seigneurs justiciers et avec la justice royale ${ }^{30}$. Ces quelques thèmes prouvent

" [...] home emprisonné ne doit responce, car il est despoillé de sa personne "; enfin, toujours au tome 4 , chapitre $\mathrm{N}$ « decisions ajoutées ", paragraphe 13 , page 519 : " si aucun estoit en prinson pour debte, et il fust adjourné davant aucun juge seculier, et il y envoye son exoine de celle prinson, elle n'est pas recevable, car nul ne se exoine par telle prinson ".

28. Ibid., tome 2 , chapitre $\mathrm{F}$, huitième partie, titre II " de restituer le despoillé avant toute euvre ", paragraphe 1081, p. 409-410 : " si aucun est prins et mis en la prison, et lui estant en ladicte prinson aucun autre s'ensaisinoit d'aucune chose dont celui fust pocesseur au temps qu'il fut prins, quant celui prinsonnier seroit hors de prinson il pourroit requerre à la justice qu'elle le meist en sa pocession en quoy il estoit quant il fut prins, et auroit bonne requeste, et seroit l'autre qui seroit en saisine appellé à deffendre la court de la requeste que l'autre fait : et s'il estoit prouvé qu'il fust en pocession au temps qu'il fut prins, il seroit remis en sa pocession et puis demandera l'autre ce qu'il vouldra si riens demander lui vieult".

29. Ibid., tome 4, chapitre L, vingtième partie " des aplegemens et contraplegemens, tans simples, privilegiés, en tiers pié, en declinant, que autres : de restablissemens : de monstrées : et de la fourme de proceder esdictes choses ", paragraphe 411 " instruccions aux gardes des prinsons ", p. 312-313 : " saichent les cas de la detencion des prisonniers; ne les partent point de la chartre; enquierent s'ilz sont clercs; serchent lesdiz prisonniers et leurs bourses en leurs presences, les retiennent et baillent au juge; leur ostent leurs cousteaux et saintures; separent lesdiz prisonniers qu'ilz ne puissent parler ensemble, ne eulx entreforger; ne laissent parler nulles gens avecques eulx sans congié, et encores qu'ilz soient presens au parlement; ne les eslargissent ne remuent de lieu en autres sans congié; visitent les prisonniers criminelz quatre foiz par jour, c'est assavoir au matin, à disner, à vespres et au coucher; ne facent ne ne facent faire aucuns messaiges aux amis desdiz prisonniers; et si les serviteurs les font, ilz en respondront; es choses qui leur seront baillées pour boire, menger ou autrement, gardent s'il y a lectres, cousteaux, ne autres choses; ne laissent entrer que les gens de conseil avecques le juge quant il fera examen; ne revelent riens de l'examen ne d'autre chose qu'ilz aient ouy dire; ne baillent point de boys aux prisonniers pour eulx chaufer; bien leur peut on bailler du charbon de jours et non de nuyz ". Le texte dépeint un environnement dans lequel les détenus semblent être strictement surveillés. Par ailleurs, la coutume nous apprend que la garde des prisonniers est une charge sérieuse qui peut exposer le gardien à de lourdes sanctions en cas d'aide apportée au détenu pour qu'il s'évade, ibid., tome 1, chapitre E (1411), paragraphe 89 , p. 433 : " si celui qui est accusé de cas criminel et emprisonné pour le cas brise les prinsons et il est reprins il est atainct du cas et le peut on pugnir. Et semblablement, si celuy de qui il est en la garde luy donne faveur de s'en aller, il sera pugny d'autelle peine comme celle du malfaicteur".

30. Ibid., tome 2 , chapitre F, neuvième partie, titre III « de emprinsonner crimineulx, et les rendre à leur juge s'ilz sont requis ", paragraphe 1310, p. 485 : " justice qui fait suyre laron ou malfaicteur qui est prins en autre justice et celle justice le vient puis requerre, il l'auroit par la coustume du pais d'Anjou " et paragraphe 1311, p. 486 : "si aucun malfaicteur qui a fait meurtre ou larecin en la terre d'aucun baron s'en vait depuis en aucune chastellenye et il est prins, celui en qui chastellenie le meffait fut fait le doit avoir en poiant IIs VId à l'autre baron. Et si le meffait avoit esté fait en la terre d'aucun valvasseur, le valvasseur le doit avoir en rendant les IIs VId à son seigneur, pour quoy il ait varié en sa terre " ou encore paragraphe 1314, p. 487 : "si aucun malfaicteur estoit en la prinson d'aucune justice pour la suspecion d'aucun meffait, et celui prinsonnier brisast la prinson et s'en allast, et puis fust prins en autre justice pour celui fait, la justice à qui il auroit 
que la législation concernant l'emprisonnement et le statut du prisonnier était bien présente. Il reste à savoir ce que les sources conservées peuvent nous apprendre de la pratique.

\section{Prisons et prisonniers à travers les sources de la pratique : une approche sociologique}

Avant toute chose, il convient de livrer quelques chiffres sur lesquels notre article s'appuie. Au cours de notre dépouillement, nous avons rencontré 24 cotes dans lesquelles nous avons trouvé des mentions de prison ou des passages concernant les prisonniers. Bien évidemment plusieurs de ces cotes peuvent concerner un même lieu. Néanmoins, ces informations recoupées les unes avec les autres nous ont permis de dresser une carte sur laquelle figurent les lieux où, semble t-il, une prison était présente. Soulignons bien que ne figurent sur cette carte que les lieux cités dans nos sources et que par conséquent elle n'a pas de caractère exhaustif quant au nombre de prisons qu'il pouvait y avoir en Anjou au bas Moyen Âge ${ }^{31}$ (Figure 1).

Dans un premier temps, il est intéressant de se demander si la présence d'une prison est conditionnée par les droits de justice détenus par les seigneurs. En effet, ces derniers pouvaient être bas ${ }^{32}$, moyen ${ }^{33}$ ou haut-justiciers $^{34}$ et bien souvent l'importance des droits de justice détenus reflétait

brisé la prinson le devroit avoir, en poiant IIs VId à celui qui le tendroit et les despens du forfaicteur faiz par avant qu'il l'eust requis, et non pas cieulx par après. Mais si celle justice estoit souveraine de l'autre qui tenist de luy les lieux ou la prinse auroit esté faicte, ou il estoit son subgit, n'en rendroit nulz des deux soulx sis deniers; car l'omme n'en rend nulz à son seigneur souverain ne à son subgit, mais l'en les rend de voisin à voisin ". Enfin, notons que sont abordées les relations entre la justice royale et les justices concédées, ainsi, titre XVI « de requerir et adjourner crimineulx et delinquans en leuer abscence pour ester à droit ", paragraphe 1357, p. 500 : " si aucuns justiciers tiennent personnes em prinson, et les gens du Roy commandent de par le Roy qu'il leur rendent ou baillent ceulx prinsonniers, ilz ne sont pas tenuz de la faire; car nul n'est tenu à soy dessaissir : mais ilz leur doivent ouvrir les prinsons, et si iceulx prennent iceulx prinsonniers, lesdiz officiers doivent venir aux gens du Roy et requerir leur droit. Et aussi peut l'en faire de moult d'autres choses".

31. Michel Le Mene, Les Campagnes angevines à la fin du Moyen Âge (vers 1350-vers 1530). Étude économique, Nantes, 1982, p. 450. Il existait des prisons permanentes seulement dans les sièges des grands ressorts comme par exemple à Craon, à Candé, à Baugé, à Rochefort. Des prisons qu'il faudrait ajouter à notre propre inventaire. Concernant nos sources nous n'avons pas pu faire cette distinction entre des prisons qui seraient d'usage occasionnel et des prisons qui seraient permanentes. De même, Céline TIXIER dans, Montreuil-Bellay, une petite ville frontière à la fin du Moyen Âge (milieu XIVe-fin $X V^{e}$ siècle), mémoire de maîtrise, université d'Angers, 2000, p. 49 note qu'à MontreuilBellay le seigneur entretient une prison, une potence et un auditoire de justice.

32. Les droits de basse justice appartenaient à d'innombrables petits seigneurs; ils n'étaient compétents que pour les délits non passibles de mort et pour les causes civiles de moindre importance.

33. La moyenne justice ne se mit en place que progressivement au cours du XIV ${ }^{\mathrm{e}}$ siècle. Les droits de moyenne justice se situent entre ceux dévolus aux bas justiciers et ceux concédés aux hauts justiciers.

34. Les droits de haute justice entraînaient une plénitude de juridiction au criminel et 
Figure 1 - Localisation des prisons citées comme relevant de justices seigneuriales en Anjou

(Sources à l'appui de cette carte : voir annexe 1)

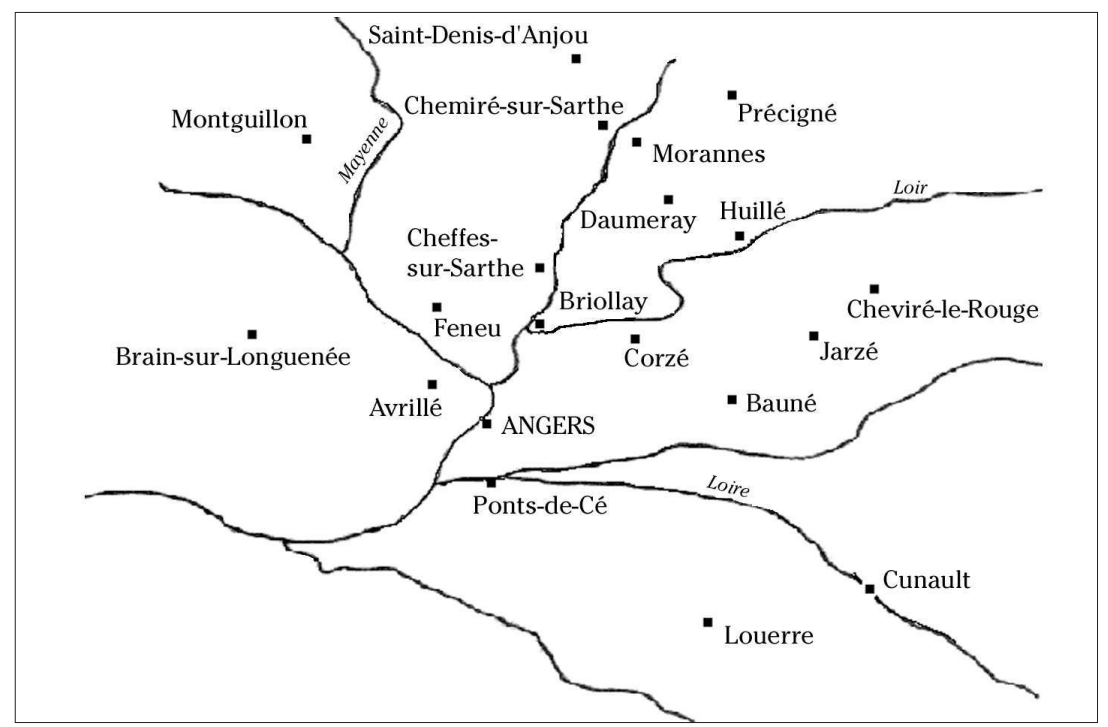

la puissance du seigneur et l'importance économique et financière de sa seigneurie. L'exercice de la justice entraîne nécessairement des coûts financiers souvent non négligeables ${ }^{35}$. Il faut rémunérer le personnel judiciaire (sénéchal, sergent, recors, parfois un geôlier...), et le cas échéant entretenir un lieu servant à la détention des prisonniers. L'obligation d'entretenir une prison et de solder du personnel à cet effet n'est pas présente dans la coutume d'Anjou. Les quelques recherches effectuées sur les droits de justice détenus par les seigneurs concernés par notre étude nous ont permis de déterminer que les seigneurs de Briollay ${ }^{36}$, Cheffes-sur-Sarthe ${ }^{37}$, Cheviré-

au civil. Les signes extérieurs sont le gibet ou fourches patibulaires. Ainsi, la force du seigneur haut justicier était qu'il pouvait condamner à mort.

35. Michel LE MENE, Les Campagnes angevines..., op. cit., p. 450 note qu'au demeurant l'action intentée contre un criminel coûtait fort cher. La location d'une prison, là où il n'en existait point de permanente, la garde du prisonnier, son entretien, son transfert à Angers, voire à Paris en cas d'appel, son retour une fois l'arrêt rendu afin qu'il soit exécuté sur les lieux du crime, le recours au bourreau, la remise en état du gibet... atteignaient souvent plusieurs dizaines de livres sans qu'on eut espoir de les récupérer.

36. MAthieu, Isabelle, Les Justices seigneuriales en Anjou et dans le Maine au bas Moyen Âge, mémoire de DEA, université d'Angers, 2002, p. 79; la baronnie de Briollay est une création de Foulques Nerra; le baron possédait la haute, moyenne et basse justice et spécialement le droit sur tous les pêcheurs, le droit de jalage sur les vins.

37. Ibidem, p. 82, ce sont les moines de Saint-Nicolas d'Angers qui ont établi un prieuré à Cheffes. Le fief du prieuré avait moyenne et basse justice. Les assises se tenaient à Cheffes dans la maison de la Barre. La seigneurie qui appartient jusqu'au XIII ${ }^{\mathrm{e}}$ siècle aux seigneurs du Lude, passe au XIV ${ }^{\mathrm{e}}$ siècle à ceux du Plessis-au-Vent (devenu au Xv $\mathrm{X}^{\mathrm{e}}$ siècle le 
le-Rouge ${ }^{38}$, Corzé ${ }^{39}$, Huillé ${ }^{40}$, Jarzé ${ }^{41}$, Morannes ${ }^{42}$, des Ponts-de-Cé ${ }^{43}$ et de Saint-Denis-d'Anjou ${ }^{44}$ étaient haut-justiciers. On entrevoit donc l'existence d'un possible rapport entre d'un côté les droits de justice revendiqués et de l'autre la nécessité et la possibilité financière de détenir une prison et de rendre la justice à la hauteur des droits affichés. Par ailleurs, un seigneur bas-justicier, de par la nature même de ses droits de justice, était immanquablement moins exposé à des délits importants nécessitant un emprisonnement qu'un seigneur haut justicier.

Plessis-Bourré). La seigneurie relevait de Briollay et obtient à une date indéterminée le titre de châtellenie.

38. Ibid., p. 83, en vertu de l'acte de fondation de 1040, le prieuré possédait la seigneurie, maison forte, moulins, fours banaux, haute, moyenne et basse justice, droit de pêche, carcan et fourches patibulaires.

39. Ibid., p. 91, la seigneurie appartient au Xve siècle au seigneur de Jarzé (haut justicier) et l'on voit en 1482 le curé de Corzé rendre hommage à Jean Bourré. Les droits du seigneur de Jarzé étaient contestés par le seigneur de Chement qui prétendait posséder les droits honorifiques attribues au fondateur de l'église. La vente en 1452 par le seigneur de Jarzé au seigneur de Chement chanoine de la cathédrale d'Angers, du fief qu'il possédait à Corzé calma toute contestation quant aux droits honorifiques. Le seigneur de Jarzé continue de s'intituler seigneur de Corzé jusqu'au XVIII ${ }^{\mathrm{e}}$ siècle.

40. Ibid., p. 100, l'abbaye Saint-Serge d'Angers établit un prieuré à Huillé. Le prieur avait droit de haute justice et de garenne. Juste à coté de la seigneurie du prieuré, il y avait une seigneurie d'Huillé. La séparation entre les deux lieux était matérialisée par le ruisseau d'Amours. La seigneurie d'Huillé relevait de la baronnie de Châteauneuf.

41. Arch. dép. de Maine-et-Loire, 8J14. Ce registre d'assises, de par les affaires exposées, montre que le seigneur était haut justicier (notamment des litiges concernant des droits de chasse et des droits de justice).

42. MAthieu, Isabelle, Les justices seigneuriales..., op. cit., p. 116, Morannes fait partie dès l'origine de la dotation de l'Évêché d'Angers. Les domaines de l'évêque formaient une châtellenie dont le sénéchal rendait la justice au nom de ce dernier. Des déclarations rendues aux assises et des censives font état de l'union à la châtellenie de Morannes de la baronnie de Gratecuisse en 1432. Tout laisse supposer que le seigneur, compte tenu de l'importance de sa seigneurie, détenait des droits de haute justice. Par ailleurs, une affaire relative à une mort suspecte, délit relevant normalement de la haute justice, a été retrouvée dans le registre d'assises G 152 au f ${ }^{\circ} 46$ : " Jehan Collasseau 15 s finés pour un deffaut de soy rendre d'office où il estoit pour le cas que l'on disoit contre luy qu'il avoit esté cause participant et consentant de la mort de Jehan de Marigné et envoyé au principal sans riens jugez en cause et sauf à le faire revenir où cas qu'il seroit trouvé par informacion ou autrement deuement qu'il en eust esté couppable ".

43. Arch. dép. de Maine-et-Loire, $\mathrm{H} 83, \mathrm{f}^{\circ} 71 \mathrm{v}^{\circ}:$ : [...] que ledit Bouget a servy mort comme d'estre pendu et estranglé à la justice de la fourest Saint-Aulbin... ". La sentence ici présente, à savoir la condamnation à mort, accrédite le fait que le seigneur devait être haut justicier.

44. Froger, Arnaud, La Reconstruction d'une seigneurie du chapitre cathédral d'Angers. Saint-Denis-d'Anjou et Chemiré-sur-Sarthe (1441-1516), mémoire de maîtrise, Université d'Angers, 2000, p. 56 note que " le chapitre Saint-Maurice avait droit de haute, moyenne et basse justice sur ses terres de Chemiré et de Saint-Denis ". Par ailleurs, des procès conservés pour Saint-Denis montrent clairement que le seigneur utilisait pleinement ses droits de haute justice en prononçant des peines de mort : Arch. dép. de Maine-et-Loire, $\mathrm{G} 575, \mathrm{f}^{\circ} 248 \mathrm{v}^{\circ}$ : « [...] pour les cas par luy commis soit condempné à mort d'estre pendu et estranglé à la justice patibulaire de la terre et seigneurie de Saint-Denis-d'Anjou... ". 
Concrètement, nous avons eu la chance de retrouver plusieurs registres dans lesquels sont relatées des affaires occasionnant l'emprisonnement des délinquants mis en cause. Il a été possible de réunir un corpus de 39 individus ${ }^{45}$ dont 5 femmes qui, pour différents motifs et pour des périodes de durée variable, ont passé un moment en prison. Ainsi, Gillet Veillon et Jean Bouget sont " prinsonniers à presens detenuz en noz prinsons du Pont de Sée ${ }^{46}$ ". De même, Perrin Lesuor et Michau Durée " sont demourés prisonniers en prison " pour divers vols de vin, de céréales et de linge ${ }^{47}$. "Jehan Chopin accusé d'avoir tendu, thezuré et prins connilz et autres gibiers au dedens de la terre et seigneurie dudit lieu de Seaulx et de la Fillotiere " est aussi emprisonné mais l'affaire ne nous en apprend pas plus sur la durée de la détention et l'issue du procès ${ }^{48}$. Le registre de SaintDenis-d'Anjou contient neuf procès au cours desquels nous découvrons les interrogatoires des prisonniers, huit hommes et une femme ${ }^{49}$. Cette liste pourrait être allongée par d'autres exemples ${ }^{50}$, néanmoins il nous semble

45. Ces 39 individus apparaissent dans 30 affaires. La différence provient du fait que plusieurs individus peuvent être impliqués dans une même affaire. Par exemple, Arch. dép. de Maine-et-Loire, $1^{\mathrm{E}} 1174, \mathrm{f}^{\circ} 30$ : "Guillaume le Gentilhomme, Jehan Gaudin, Jehan Mirondet sur ce que l'on dit contre eulx qu'ilz ont couppé, prins, emporté ung chesne des boys de la court ce qu'ilz ne povoient ne devoient faire pour lequel cas ilz furent mis es prisons deceans...".

46. Arch. dép. de Maine-et-Loire, $\mathrm{H} 83$, du f ${ }^{\circ} 61$ au f ${ }^{\circ} 71$. Les deux individus sont mis en cause pour divers vols (moutons, argent, linge, outils, nourriture...). L'interrogatoire de Gillet Veillon commence le 13 octobre 1482 et s'achève le 13 novembre 1482 par la condamnation suivante " [...] en ce sur ce l'avons et opinion de plusieurs gens de conseil clercs et autres et consideré ce que à veoir et consideré en la matiere nous par notre sentence juge avons condamné et condampnons ledit Gillet Veillon à estre batu tout neu avecques fouez à la justice de la fourest et oultre est bany hors ladite terre de Saint-Aulbin du Pont de Sée et de la fourest Saint-Aulbin... ". Celui de Jean Bouget commence à la même date et se termine le 18 novembre 1482 par la condamnation suivante " comme d'estre pendu et estranglé à la justice de la fourest Saint-Aulbin ". Il ressort que la détention a été brève puisque l'affaire fut réglée en un peu plus deux mois.

47. Arch. dép. de Maine-et-Loire, $1 \mathrm{Hs}$ B176, f $110 \mathrm{v}^{\circ}$. L'emprisonnement a lieu dans la prison de la seigneurie d'Egrefoin (Aigrefoin, village qui s'étend sur les communes de Brain-sur-l'Authion et du Plessis-Grammoire, vu l'imprécision des renseignements cette localité ne figure pas sur cette carte), terre dépendant des biens de l'Hôpital Saint-Jean, malheureusement il n'y a pas de détail concernant le temps de la détention et l'issue définitive de l'affaire (relaxe, peine pécuniaire, corporelle...?).

48. Arch. dép. de Maine-et-Loire, $1^{\mathrm{E}} 1174, \mathrm{f}^{\circ} 42$ et f ${ }^{\circ} 42 \mathrm{v}^{\circ}$, l'affaire est inscrite à l'année 1489.

49. Arch. dép. de Maine-et-Loire, G575 (1501-1511). Nous découvrons successivement les interrogatoires de Grégoire le Taillandier (empoisonnement), Guillemine la Robelotte (empoisonnement), Jacquet le Corvasier (vols, complice dans les empoisonnements), Michau Jouenneaux (non précisé), Jean Brulle (vols divers), Mathurin Gruau (sorcellerie), André Pineau (vol d'un cheval), Jean Pelart (vols divers), Michel Priet (divers vols) tous détenus " ès prisons deceans".

50. Arch. dép. de Maine-et-Loire, $1^{\mathrm{E}} 1174$, f ${ }^{\circ} 30$ (1474), Guillaume le Gentilhomme, Jean Giraudin et Jean Mirondet sont mis en prison pour avoir volé du bois appartenant à la court; 15 G 19, $\mathrm{f}^{\circ} 28$ (1454), Jeanne veuve de feu Jean Chauveau est suspectée d'infanticide et retenue prisonnière; $\mathrm{f}^{\circ} 31 \mathrm{v}^{\circ}(1467)$, Jean Crespon et Jean Arnoul sont mis en prisons pour violences physiques à l'encontre de Pierre Chenu; f 170 (1461-1467), Pierre Lecamus est constitué prisonnier pour " plusieurs exces, delitz "; f $192 \mathrm{v}^{\circ}$ (1473), 
intéressant de nous arrêter sur une affaire assez particulière et, avouons le, bien amusante relatant l'arrestation d'un individu qui est sommé, sur les ordres du sergent de se rendre en prison, ce qu'il ne fait pas :

«Perrin Bonneau deffaut et sera prins au corps sur ce que l'en dit contre lui que certain blé avoit esté prins ou povoir deceans par feu Perrin Bonneau ledit bonneau a esforcé de gens armez d'armes invasibles fist saier ledit blé tout vert et en fist emporter partie hors de notre povoir et non obstant ledit Bonneau a prins et emporté ledit blé qui estoit saisi comme dit est sans congié de personne qui eust povoir et pour ce notre dit sergent lui commanda se rendre en noz prinsons et lui bailla le chemin pour prison lequel ne se y rendit aucunement ${ }^{51}$."

Les protagonistes et leurs délits étant présentés, il faut se pencher sur l'issue finale de ces affaires. À ce propos, notre documentation se scinde vraiment en deux ensembles; d'un coté nous avons les registres de SaintDenis-d'Anjou et des Ponts-de-Cé pour lesquels sont conservés les procès dans le détail et par conséquent les informations sont précises et nombreuses (ce sont, semble t-il, des affaires qui étaient jugées assez importantes pour que soit diligentée une enquête très méticuleuse), de l'autre nous avons tout un groupe d'affaires où le personnel judiciaire passe aussi vite sur les circonstances des faits que sur la présentation des individus et les sentences prononcées à l'issue de l'emprisonnement. Cette dichotomie se retrouve clairement dans les peines prononcées. En effet, les onze individus concernés dans les procès de Saint-Denis et des Ponts-de-Cé connaissent les peines les plus lourdes : trois hommes sont pendus et étranglés ${ }^{52}$, un est battu ${ }^{53}$, la femme est brûlée ${ }^{54}$, deux hommes sont fustigés et

Macé Falardeau est emprisonné car il a refusé d'obéir aux ordres du sergent; $\mathrm{f}^{\circ} 196 \mathbf{v}^{\circ}$ (1473), Guillaume Bereau est emprisonné pour " abus et tromperies "; f 197 (1473), c'est Clément Rouxigneul qui est incarcéré pour " ses merites ". G 443, f 37 v (1472), Jamin Charbonnier, Perrin Charbonnier, Julliot Charbonnier, Jean Baugeays, Pierres Charbonnier, Gervais Bureau pour des vols de bois sont condamnés à rester en prison jusqu'à ce qu'ils aient réparé financièrement leur délit. G 891, premier parchemin (1358), mention d'un certain monseigneur Guillaume Dusages prisonnier. 8 J 63, deuxième registre, $\mathrm{f}^{\circ} 90$ (1492), " Jamet Bouridart en la demande que on luy fait pour avoir iniurié en jugement les assises tenues les officiers de la court en disant qu'ilz n'estoient que fouerouzeaux et quoquineaux et plusieurs autres parolles iniurieuses et deshonestes et ne le peult denyez pour ce qu'il sera prouvé à suffire contre luy par Eustache Lasnon et autres plusieurs et pour ce le procureur de la court conclud contre luy qu'il soit condempné et puis contrainct reparez et amender lesdites iniures à monseigneur le senechal procureur et autres officiers de la court d'amende honorable et prouffitable telle qu'il apprendra par raison et à tenir prin-

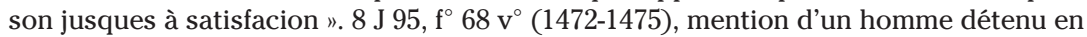
prison et exoiné par sa femme. $8 \mathrm{~J} 14, \mathrm{f}^{\circ} 94$, (1494), Guillaume Jarry tient prison pour "batures, exces et larecins ", $\mathrm{f}^{\circ} 102$ (1496), Guillemine la Molle a connu la prison pour divers " fur et larecin "; $\mathrm{f}^{\circ} 168 \mathrm{v}^{\circ}$ (1498), Jean Nouschet est incarcéré pour avoir " chacé de nuyt tendu et trezuré et prins congnilz ou parc de monseigneur "; f $260 \mathrm{v}^{\circ}(1500)$, Jeanne la Bidaude est arrêtée et emprisonnée pour avoir volé du blé au moulin de la cour.

51. Arch. dép. de Maine-et-Loire, $\mathrm{H} 22, \mathrm{f}^{\circ} 44 \mathrm{v}^{\circ}$ (1411).

52. Arch. dép. de Maine-et-Loire, $\mathrm{H} 83, \mathrm{f}^{\circ} 71 \mathrm{v}^{\circ}$ et $\mathrm{G} 575, \mathrm{f}^{\circ} 55 \mathrm{v}^{\circ}, \mathrm{f}^{\circ} 153 \mathrm{v}^{\circ}$.

53. Arch. dép. de Maine-et-Loire, $\mathrm{H} 83, \mathrm{f}^{\circ} 66$.

54. Arch. dép. de Maine-et-Loire, G 575, f ${ }^{\circ} 109$. 
condamnés à rendre les biens dérobés ${ }^{55}$, un est fustigé, essorillé et banni à perpétuité ${ }^{56}$, un est battu et banni ${ }^{57}$, un autre est banni à perpétuité ${ }^{58}$, enfin il y a un individu pour lequel nous ne connaissons pas le châtiment ${ }^{59}$. Concernant les dix-neuf affaires restantes, dans six cas les affaires se soldent par des remises en liberté grâce à un cautionnement ${ }^{60}$, dans cinq cas une amende accompagne la relaxe des individus ${ }^{61}$, dans deux cas les personnes sont relâchées car rien n'a pu être prouvé contre elles ${ }^{62}$. L'issue des six cas restants est la suivante : demeuré en prison ${ }^{63}$, demeuré en prison jusqu'au paiement d'une amende ${ }^{64}$, relâché et les biens saisis ${ }^{65}$, procès transféré vers un autre siège ${ }^{66}$, le père a été diligenté pour réprimer sa fille mise en cause ${ }^{67}$, l'issue n'est pas connue ${ }^{68}$. Dans l'ensemble de nos affaires la prison ne représente donc bien qu'une étape vers les peines définitives qui sont prononcées.

\section{Les conditions d'incarcération et d'évasion}

Par ailleurs, il a été possible, grâce aux procès de Saint-Denis ${ }^{69}$, des Pontsde-Cé ${ }^{70}$ et de Sceaux ${ }^{71}$, de donner quelques détails concernant les délinquants incarcérés. Ce corpus de 12 prisonniers ${ }^{72}$ nous livre des informations, à travers leurs interrogatoires respectifs, sur leur âge, leur lieu de naissance et de résidence, parfois leur métier et leur situation de famille (Tableau 1).

D'extraction sociale modeste et rurale, nos individus ne sont ni très jeunes ni très vieux (la majorité a entre 20 et 30 ans), souvent mariés et ont parfois des enfants; voilà les quelques points communs qui existent entre eux. Notons qu'ils n'affichent pas un profil ou des caractéristiques bien spécifiques.

55. Arch. dép. de Maine-et-Loire, G 575, $\mathrm{f}^{\circ} 56 \mathrm{v}^{\circ}, \mathrm{f}^{\circ} 219 \mathrm{v}^{\circ}$.

56. Arch. dép. de Maine-et-Loire, G 575, $\mathrm{f}^{\circ} 253 \mathrm{v}^{\circ}$.

57. Arch. dép. de Maine-et-Loire, G 575, f ${ }^{\circ} 142$.

58. Arch. dép. de Maine-et-Loire, G 575, f ${ }^{\circ} 236$.

59. Arch. dép. de Maine-et-Loire, G 575, du f 176 au f $^{\circ} 183 \mathrm{v}^{\circ}$.

60. Arch. dép. de Maine-et-Loire, G 891, premier parchemin; $8 \mathrm{~J} 95, \mathrm{f}^{\circ} 68 \mathrm{v}^{\circ} ; 1^{\mathrm{E}} 1174, \mathrm{f}^{\circ} 30$ et $\mathrm{f}^{\circ} 42 \mathrm{v}^{\circ} ; 15 \mathrm{G} 19, \mathrm{f}^{\circ} 170$ et $\mathrm{f}^{\circ} 196 \mathrm{v}^{\circ}$.

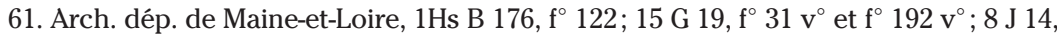
$f^{\circ} 94 \mathrm{v}^{\circ}$ et $\mathrm{f}^{\circ} 102$.

62. Arch. dép. de Maine-et-Loire, 15 G 19, f $^{\circ} 28 ; 8 \mathrm{~J}$ 63, deuxième registre, $\mathrm{f}^{\circ} 90$.

63. Arch. dép. de Maine-et-Loire, $1 \mathrm{Hs}$ B $176, \mathrm{f}^{\circ} 110$.

64. Arch. dép. de Maine-et-Loire, G 443, $\mathrm{f}^{\circ} 37 \mathrm{v}^{\circ}$.

65. Arch. dép. de Maine-et-Loire, 15 G 19, f 197.

66. Arch. dép. de Maine-et-Loire, $8 \mathrm{~J} 14, \mathrm{f}^{\circ} 168 \mathrm{v}^{\circ}$.

67. Arch. dép. de Maine-et-Loire, $8 \mathrm{~J} 14, \mathrm{f}^{\circ} 260 \mathrm{v}^{\circ}$.

68. Arch. dép. de Maine-et-Loire, $\mathrm{H} 22, \mathrm{f}^{\circ} 44 \mathrm{v}^{\circ}$.

69. Arch. dép. de Maine-et-Loire, G 575.

70. Arch. dép. de Maine-et-Loire, H 83.

71. Arch. dép. de Maine-et-Loire, $1^{\mathrm{E}} 1174, \mathrm{f}^{\circ} 42$ et f $\mathrm{f}^{\circ} 42 \mathrm{v}^{\circ}$.

72. Ce corpus peut sembler bien maigre mais sur l'ensemble de notre documentation, ce sont les seuls individus pour lesquels nous avons autant de renseignements. On ne peut que constater et regretter les lacunes des sources et se contenter de ce qu'elles nous livrent. 
Tableau 1 - Identité des prisonniers

\begin{tabular}{|l|l|}
\hline 1E1174 & $\begin{array}{l}\text { Jean CHOPIN, } 30 \text { ans, né à Pouancé, vit depuis } 8 \text { ans dans le bourg de } \\
\text { Sceaux, maréchal }\end{array}$ \\
\hline H83 & Gillet VELLLON, 20 ans, né aux Ponts-de-Cé, vit aux Ponts-de-Cé \\
\hline H83 & Jean BOUGET, 30 ans, né à Saint-Pierre d'Angers, marié, des enfants \\
\hline G575 & $\begin{array}{l}\text { Grégoire LE TAILLANDIER, } 42 \text { ou } 43 \text { ans, né à Saint-Denis-d'Anjou, } \\
\text { marié, des enfants }\end{array}$ \\
\hline G575 & Guillemine LA ROBELOTTE, 25 ans, mariée deux fois \\
\hline G575 & Jacquet LE CORVAISER \\
\hline G575 & Michau JOUENNEAUX \\
\hline G575 & Jean BRULLE, 26 ans \\
\hline G575 & Mathurin GRUAU \\
\hline G575 & $\begin{array}{l}\text { André PINEAU, } 42 \text { ans, né à Villers-Charlemagne dans le diocèse du Mans } \\
\text { s'installe paroisse de Ruillé, puis ensuite à Chemiré-sur-Sarthe, labou- } \\
\text { reur de vigne, conduit les harnois, marié }\end{array}$ \\
\hline G575 & Jean PELART, 27 ans, né à Saint-Denis-d'Anjou, laboureur, homme de bras \\
\hline G575 & $\begin{array}{l}\text { Michel PRET, } 35 \text { ans, né à de Brissarthe, } \\
\text { s'installe par la suite à Saint-Denis, laboureur, homme de bras, marié }\end{array}$ \\
\hline
\end{tabular}

Par ailleurs, il est possible d'appréhender des donnés concernant directement leurs déboires avec la justice. Ainsi, mis à part le récit des délits et les sentences, leurs antécédents sont soigneusement retranscrits de même que leurs tentatives d'évasion (voir Annexe 2). La durée des incarcérations n'est pas mentionnée cependant nous pouvons remarquer que les procès sont rondement diligentés, s'étalant de quelques semaines à quelques mois ${ }^{73}$. Les détentions sont rythmées par des mises à la question justifiées par le personnel judiciaire pour accéder pleinement à la vérité. Ainsi, trois hommes ${ }^{74}$ et une femme ${ }^{75}$ subissent cette épreuve d'une violence inouïe

73. Roger GRAND aborde la question de la rapidité des jugements à rendre dans « La prison et la notion d'emprisonnement dans l'ancien droit ", Nouvelle revue..., op. cit., p. 69. Ainsi, à partir d'un exemple, il note « les baillis se servaient même de la prison préventive avec une telle désinvolture, y laissant languir outre mesure les prévenus, que le roi, par mandements réitérés, fut obligé de leur rappeler le devoir qu'ils avaient de juger au plus vite les prisonniers pour les condamner ou les renvoyer quittes".

74. Arch. dép. de Maine-et-Loire, $\mathrm{H} 83, \mathrm{f}^{\circ} 70$ : « [...] lequel cas il (Jean Bouget) a nyé et partant mis en question extraordinaire... "; G $575, \mathrm{f}^{\circ} 14:$ " [...] veues lesquelles variacions denegacions et confrontacion nous avons presenté audit prinsonnier (Grégoire le Taillandier) la question extraordinaire en le mectant sur ung bain ataché à une corde par les piedz par bas et par hault par dessoubz les esselles et luys avons baillé les gresillons aux posses et avant que luy faire aucune violence ne torture à sa personne luy avons remonstré les denegacions... ", $\mathrm{f}^{\circ} 51 \mathrm{v}^{\circ}:$ "veues lesquelles confrontacions proces et confessions et presumpcions avons apoincté que ledit acquet le Corvasier sera mis en question extraordinaire pour en scavoir plus amplement la verité par sa bouche et que notredit apoinctement sera par nous executé et fait executer comme il apartiendra ".

75. Arch. dép. de Maine-et-Loire, G 575, Guillemine est plusieurs fois, au cours de son emprisonnement, mise à la question ainsi à Saint-Denis, $\mathrm{f}^{\circ} 52 \mathrm{v}^{\circ}:$ : veues lesquelles denegacions proces et confrontacions avons apoincté et declaré que ladite Guillemine sera mise en gehenne et question extraordinaire pour en scavoir plus amplement la verité de 
mais légale ${ }^{76}$. De ces quatre individus impliqués dans des affaires jugées assez graves pour qu'ils soient mis à la question, trois vont s'évader pendant leur détention. Ainsi le larron Jehan Bouget « brise " les prisons ${ }^{77}$. De même, notre couple d'empoisonneurs s'évade habilement de leur geôle ${ }^{78}$. Le récit de l'évasion ${ }^{79}$ de Guillemine la Robelotte est conservé dans son procès :

«Et premier interrogée ladite Robelote par serment quy l'a mené de sortir et briser lesdites prinsons et s'enfouyr au lieu de Auvers le Hamon ou pays du Maine où elle a esté reprinse après ledit bris et eschapement desdites prinsons et quy luy a donné confort et aide à sortir d'icelles prinsons.

Dit et respond que ung nommé Michau Trochon luy a donné confort et aide à sortir d'icelles prinsons lequel Trochon pour la tirer de la prinson où ladite Robelote estoit rompit une terrasse estant entre deux coulombes ou en l'une d'icelles coulombes estoit appousée une chaigne de fer à laquelle tenoient les fers dont estoit enferrée ladite Robelote.

Aussi dit et confesse que iceluy soir que ledit Trochon rompit ladite terrasse une heure avant que ladite Robelote sortist iceluy Trochon luy bailla une lime pour soy defferrer avecques laquelle lime elle lima la goupille desdits fers esquelx elle estoit enferrée par le conseil dudit Trochon quy luy conseilla ainsi le faire.

Et luy dist ledit Trochon qu'il ne luy toucheroit point et qu'elle sortist par la rompture de ladite terrasse et que touz les huys des pressouers et celiers de la maison de cyens estoient touz ouvers et qu'elle ne trouveroit aucun destourbier ne empeschement et qu'elle n'eust nulle paour et que pour sortir par le bas desdites maisons ou sont les pressouers de messieurs il n'y

sa bouche " mais aussi lors de son appel à Paris, $\mathrm{f}^{\circ} 95 \mathrm{v}^{\circ}$ et $\mathrm{f}^{\circ} 96$ : lequel Pagerit l'a menée en la chambre dudit fort Levesque et en la presence de nous l'a deserpillée de sa robbe mise en sa cocte assise sur la pierre lyée et actachée l'une main après l'autre à deux boucles de fer et en icelle actachant a protesté icelle Guillemine que quelque chose qu'elle diroit en icelle question qu'elle s'en desdiroit après, luy ont esté actacher les piez levée de dessus terre et soubs les cordes apposé ung treteau et ce fait par ledit Pagerit questionner dessusdit luy a esté baillée une serviette et de l'eaue à avaler jusques à la quantité d'une pincte ou environ et pour ce qu'elle ne sonnoit mot luy en a esté donné encores autant et a prié et requis qu'on cessast...

76. Nicole GONTHIER dans " La violence judiciaire à Dijon à la fin du Moyen Âge ", Mémoires de la société pour l'histoire..., op. cit., p. 27, souligne que la torture apparaît bien comme une des violences majeures de l'instruction. Il semble qu'elle soit parfois "médicalisée " c'est-à-dire que l'on prenne soin de ne pas aller au-delà des forces du supplicié.

77. Arch. dép. de Maine-et-Loire, $H 83, \mathrm{f}^{\circ} 88 \mathrm{v}^{\circ}$ : « dit que dimanche darrenier passé, il eschappa des prinsons et luy aida à s'en tirer Jehan Moreau et Yvonnet Peu et d'illecques s'en alla à Fremur en ung paillier et y dormit toute la nuyt et s'en alla de là en une eglise et dit qu'il oyt la messe et puis s'en vint en ceste ville d'Angers ou il fut prins sur le pavé et mis es prinsons de la Mairie et de là fut rendu en la chartre de monseigneur le senechal d'Aniou et en laquelle il coucha une nuyt et depuis le lendemain rendu à la court deceans parce qu'ilz furent informez qu'il avoit esté constitué prisonnier par la court deceans et qu'il avoit rompu lesdites prinsons et s'en estoit allé ".

78. Arch. dép. de Maine-et-Loire, G 575, f $\mathrm{f}^{\circ} 49$ : « enquis ledit Gregoire le Taillandier quy la meu ceste nuyt passée d'avoir rompu et brisé les prinsons s'estre defferré et s'en allé hors desdites prinsons, dit qu'il a osté ses fers hors de ses jambes parce qu'il n'estoient pas bien rivez ne clouez et quant ilz ont esté ostez il s'est gecté par une fenestre et s'en est fouy et s'en fust allé loing n'eust esté maistre Pierre Bellenger et autres quy l'ont poursuyvy et ramené" ".

79. Arch. dép. de Maine-et-Loire, G $575, \mathrm{f}^{\circ} 57 \mathrm{v}^{\circ}$ et $\mathrm{f}^{\circ} 58$. 
auroit ame quy la veist ne quy en oyst riens parce qu'il n'y a nulles maisons par le bas desdits pressouers et estoit comme environ l'heure de neuf heures de nuyt ou plus et comme icelle Robelote fut hors desdites prinsons pressouers et celiers la fist passer ledit Trochon par sur ung petit mur faisant la clouason de lerre de chapitre ou en ung coing d'icelle herre y avoit de grosses pierres les unes sur les autres par sur lesquelles ledit Trochon la fist passer.

Aussi dit que en sortant desdites prinsons et pour s'en aller comme luy avoit dit ledit Trochon elle trouva touz les huys desdits seliers et pressouers ouvers et s'en sortit bien à son aise par sur ledit mur avecques l'aisde que luy faisoient lesdites pierres."

La complicité de Michau Trochon a été visiblement nécessaire pour mener à bien cette aventure. Par ailleurs, l'organisation soigneusement préparée et le parcours de cette évasion transparaissent clairement dans ce récit très détaillé.

Enfin, c'est aussi le cas de Jean Chopin qui dépose s'en être allé des prisons de Vérigné car "l'uis de ladite prison était ouvert ${ }^{80}$ ". Il est intéressant de noter comment certains de nos prévenus se défaussent de leurs actes en rapportant que la prison était restée ouverte ou que les fers qui servaient à les attacher dans leur cellule étaient mal serrés. Néanmoins, même s'il y a bien eu négligence de la part des gardes ${ }^{81}$, ils n'ont pas eu de peine à tous les rattraper dans leur fuite et dans un laps de temps très bref ${ }^{82}$.

Les hommes n'étaient pas les seuls susceptibles d'être emprisonnés en cas de délits. En effet, les sources nous livrent de nombreuses affaires ${ }^{83}$ où ce sont les animaux ${ }^{84}$ qui sont privés de liberté ${ }^{85}$ :

80. Arch. dép. de Maine-et-Loire, $1^{\mathrm{E}} 1174, \mathrm{f}^{\circ} 42$.

81. Annik PORTEAU-BITKER souligne que les prisons ne sont pas toujours des édifices bien clos et que la surveillance laisse souvent à désirer et les obstacles qui mènent à la liberté sont donc faciles à franchir et, en fait, souvent franchis; pour tenter de mettre un terme aux trop fréquentes évasions, on prend l'habitude d'attacher les prisonniers à des " ceps " ou " fers ", "L'emprisonnement dans le droit laïque du Moyen Âge ", Nouvelle revue..., op. cit., p. 239-240.

82. Nicole GonTHIER dans "Prisons et prisonniers à Lyon aux XIV et XVe siècles ", Mémoires de la société pour l'histoire..., op. cit., p. 28, conclut en notant que la prison n'est qu'un épisode assez bref entre le délit et la décision de justice et surtout [comme le constate Bernard Guénée pour le bailliage de Senlis] la prison est une éventualité normale de l'existence et ne brise aucune carrière, aucune respectabilité.

83. Arch. dép. de Maine-et-Loire, $190 \mathrm{~J} 5, \mathrm{f}^{\circ} 108 \mathrm{v}^{\circ} ; 1 \mathrm{Hs}$ B $87, \mathrm{f}^{\circ} 77 ; 1 \mathrm{Hs}$ B $176, \mathrm{f}^{\circ} 35 ; \mathrm{H} 555$, $\mathrm{f}^{\circ} 17, \mathrm{f}^{\circ} 19 \mathrm{v}^{\circ} ; \mathrm{H} 1056, \mathrm{f}^{\circ} 32, \mathrm{f}^{\circ} 33, \mathrm{f}^{\circ} 34 \mathrm{v}^{\circ}, \mathrm{f}^{\circ} 38 ; 1^{\mathrm{E}} 818, \mathrm{f}^{\circ} 20, \mathrm{f}^{\circ} 21 ; 34 \mathrm{~J} 91, \mathrm{f}^{\circ} 28 \mathrm{v}^{\circ}, \mathrm{f}^{\circ} 50 \mathrm{v}^{\circ}$; $15 \mathrm{G} 19, \mathrm{f}^{\circ} 21, \mathrm{f}^{\circ} 74, \mathrm{f}^{\circ} 120 \mathrm{v}^{\circ}, \mathrm{f}^{\circ} 151 \mathrm{v}^{\circ} ; 8 \mathrm{~J} 62, \mathrm{f}^{\circ} 47 \mathrm{v}^{\circ}, \mathrm{f}^{\circ} 52 \mathrm{v}^{\circ} ; 8 \mathrm{~J} 63, \mathrm{f}^{\circ} 31 \mathrm{v}^{\circ} ; 8 \mathrm{~J} 14, \mathrm{f}^{\circ} 159 \mathrm{v}^{\circ}$, $\mathrm{f}^{\circ} 229 \mathrm{v}^{\circ}, \mathrm{f}^{\circ} 230, \mathrm{f}^{\circ} 230 \mathrm{v}^{\circ}, \mathrm{f}^{\circ} 231, \mathrm{f}^{\circ} 248, \mathrm{f}^{\circ} 255, \mathrm{f}^{\circ} 255 \mathrm{v}^{\circ}, \mathrm{f}^{\circ} 257, \mathrm{f}^{\circ} 258 \mathrm{v}^{\circ}$.

84. Annik PORTEAU-BITKER dans "Criminalité et délinquance féminines dans le droit pénal des XIII et XIV ${ }^{\mathrm{e}}$ siècles ", Revue historique de droit..., op. cit., p. 16 évoque le fait que des procès sont intentés à des animaux, à des cadavres, à des enfants, à des fous... De même, Robert MUCHEMBLED dans Le temps des supplices, Paris, 1992, p. 42 souligne que dans des cellules souvent surpeuplées, peuvent séjourner à l'occasion des animaux; ainsi, dix chevaux sont emprisonnés deux jours à partir du 10 janvier 1409, suite à une "dette connue " de leur maître.

85. AGNEL, Émile, Curiosités judiciaires et historiques du Moyen Âge, procès contre les animaux, Paris, 1858, p. 6 « Au Moyen Âge on soumettait à l'action de la justice tous les faits 
"Jehan le Roux, Pierres Rousseau, Jehan Faullet, Guillaume Eschart, Jehan Cerneau le jeune sur ce qu'on dit contre eulx et chacun d'eulx qu'ilz font coucher leurs pourceaux en la halle et de jour n'ont autre estable tellement la halle en est enpullantée et infecte [...] en icelle maniere que les marchans et bouschers ne autres gens n'y pevent habitez honnestement et y ont esté prins les pourceaux et menez es prisons de ceste ville et delivrez avecques pleges ${ }^{86}$."

L'incarcération est souvent de courte durée et les animaux sont restitués à leurs propriétaires lorsque ces derniers se sont acquittés d'une amende ou ont fourni une caution morale (plège) ${ }^{87}$. Les propriétaires s'adonnent à un délit courant en " brisant les prisons de la court " dans le cas d'une récupération illicite des animaux mis sous séquestre ${ }^{88}$. Ainsi, "Macé Debailler sur dommage de bestes et sur rompu les prinsons de monseigneur en prenant ses bestes qui avoient esté mises en prinson et prinses es dommaines de monseigneur es vignes ${ }^{89}$ ". On entrevoit toute l'importance qu'avait la protection des biens et des terres surtout lorsque le propriétaire était le seigneur. Il s'agit de maintenir l'harmonie entre les différents troupeaux qui coexistent les uns à côté des autres, de responsabiliser les propriétaires et de rappeler et réaffirmer les prérogatives seigneuriales.

À l'issue de cette étude, il est somme toute très regrettable que sur l'ensemble de notre documentation nous n'ayons pas trouvé de description

condamnables de quelque être qu'ils fussent émanés, même les animaux. L'histoire de la jurisprudence nous offre à cette époque de nombreux exemples de procès dans lesquels figurent des taureaux, des vaches, des chevaux, des porcs, des truies, des coqs, des rats, des mulots, des limaces, des fourmis, des chenilles, sauterelles, mouches, vers et sangsues ". Les animaux n'étaient donc pas exempts de répondre de leurs faits et gestes devant les tribunaux! Dans les cas qui nous intéressent, la justice seigneuriale cherchait davantage à atteindre les propriétaires des " animaux récalcitrants " pour qu'ils veillent plus sérieusement au déplacement de leurs bêtes.

86. Arch. dép. de Maine-et-Loire, $8 \mathrm{~J} 14$ (Jarzé), $\mathrm{f}^{\circ} 171$, de même au f ${ }^{\circ} 229 \mathrm{v}^{\circ}$ nous pouvons lire « Jehan Naslin pour avoir endommagé avecques ses bestes les boys et couldraiz en faisant pestre et pasturer iceulx boys et couldraiz et menés icelles bestes es prisons deceans et depuis delivrées avecques le plege de Jehan Lambonneau ".

87. La coutume prévoit la possibilité d'un bref emprisonnement pour celui qui trouve des animaux divagants sur ses terres. Il faut donc se méfier dans les sources de ces cas d'incarcération qui ne rentrent pas dans notre problématique qui est bien de répertorier les prisons des justices seigneuriales. BEAUTEMPS-BEAUPRE, Charles-Jean, Coutumes et institutions..., op. cit., tome 2, chapitre F, titre XIV " de dommaige qui est fait ou advou par coulpe ", paragraphe 415, p. 462 " et par ladicte coustume cil qui treuve bestes en son blé, champ ou vigne deffensables les peut prendre et les tenir em prinson en son houstel ung jour et une nuyt, affin de les mener à justice pour avoir desdommaigement du dommaige que lesdictes bestes ont fait de celui à qui en a la garde, sans en faire restitucion avant que desdommaiger, qui ne baille plaige ou gaige du dommaige ".

88. Arch. dép. de Maine-et-Loire, G 1999 (Daumeray); un exemple d'amende pour un tel délit est conservé au $\mathrm{f}^{\circ} 7 \mathrm{v}^{\circ}$ "Guillaume Duvau $2 \mathrm{~s} 6 \mathrm{~d}$ pour estre envoyé sans jour pour ce que l'on disoit contre lui que sans le congié et licence de justice il a emmené ses bestes et mises hors de prinson ce qu'il ne povoit ne devoit".

89. Arch. dép. de Maine-et-Loire, 16 J 1 (Brain-sur-Longuenée), troisième cahier, f 25. 
précise des lieux ${ }^{90}$ servant à l'incarcération des détenus ${ }^{91}$ ainsi que des détails précis concernant les geôliers ${ }^{92}$. Nous savons simplement que les prisonniers pouvaient communiquer avec l'extérieur; ainsi, par exemple, " le Corvasier alla dire à ladite Guillemine à la prinson qu'elle ne sonnast mot ${ }^{93}$... " ou bien encore que " depuys qu'elle est en prinson ceans par plusieurs foiz ledit Gregoire son mary luy a dit à haulte voix à travers les murailles et huys desdites prinsons qu'elle ne dist mot ${ }^{94} \ldots$ ". D'après ces quelques témoignages, nous pouvons supputer que les paroles échangées se faisaient à la sauvette et à l'insu des gardes. Cependant, il semble qu'il était assez aisé d'accéder à ces lieux pour pouvoir espérer discuter avec les prisonniers. Nous rejoignons ici l'idée souvent avancée d'après laquelle les prisons au Moyen Âge n'étaient pas des lieux placés sous très haute sécurité $^{95}$. Enfin, il semblerait que le personnel judiciaire pouvait décider

90. Nicole GonTHIER dans son article "Prisons et prisonniers à Lyon aux XIV et $\mathrm{xV}^{\mathrm{e}}$ siècles ", Mémoires de la société..., op. cit., p. 15, nous rejoint sur les lacunes des sources : "étudiant les diverses manifestations de la criminalité et de la violence à Lyon aux XIV et $\mathrm{XV}^{\mathrm{e}}$ siècles, on s'étonne de l'absence de documents concernant directement les prisons et le traitement des prisonniers alors que les récits de rimes et délits divers abondent et sont scrupuleusement détaillés jusqu'à l'anecdocte ". De même, Robert Muchembled dans Le temps des supplices..., op. cit., p. 40, note que "faute de sources détaillées, l'étude des prisons médiévales se révèle particulièrement malaisée ". À souligner simplement que dans le registre d'assises de Morannes coté $\mathrm{G} 157$ au f 72 , on note la présence d'une prison située dans un château : "Le IX ${ }^{\mathrm{e}}$ jour de novembre l'an mil IIII soixante cinq Guillaume Rondeau a gagé l'amende de 60 s à la moderacion qui sur ce sera faicte par le senechal deceans pour quatre vaches et un veau qui ont esté prinses par le forestier es boys de l'evesque tailleys mandre de trois ans et amenés en prinson au chasteau lesquelles bestes appartiennent au sieur de la Millacerie dont ledit Rondeau avoit la garde ainsi qu'il a congneu, presens ad ce Guillaume Lenfant Simon Gaudon et Jehan le Bastart. À l'assise de Moranne tenue le XIIII jour de janvier IIIIc LXV, ladite amende a esté tauxée pour le cas dessusdit à la somme de Vs tournois parce que le chastelain et sergent ont raporté que ledit Guillaume Rondeau est très pouvre chargé de femme et enffans et qu'il ne tient ne possede aucuns heritaiges en la seigneurie deceans ne ailleurs ».

91. Annik PORTEAU-BITKER note que les prisons sont le plus souvent des constructions assez sommaires. Les seigneurs n'ont pas toujours les moyens d'en faire assurer la surveillance par des gens de métier et il est exceptionnel que des hommes acceptent encore, sans contrepartie, de garder les prisons seigneuriales, "L'emprisonnement dans le droit laïque du Moyen Âge ", Nouvelle revue..., op. cit., p. 238-239.

92. À une exception près car dans le registre de Morannes coté G 157, $\mathrm{f}^{\circ} 203 \mathrm{v}^{\circ}$, nous avons trouvé une allusion faisant état d'une transaction financière concernant la conduite de prisonniers : " Estienne Gaultier a finé en jugement à XXs pour deux contraltz l'un montant IIIIl Xs et l'autre montant XLs pour certains boys sis es marays de Vaulx sur laquelle somme a esté rabatu audit Gaultier XIIIs IXd pour certaines mises par lui faictes à la conduite des prinsonniers menez à Angers par le commandement de messieurs les senechal et bailly le premier contralt passé le VII ${ }^{\mathrm{e}}$ mars IIIIxx et l'autre le XXIII ${ }^{\mathrm{e}}$ jour d'avril IIII ${ }^{\mathrm{XX}} \mathrm{I}$ ".

93. Arch. dép. de Maine-et-Loire, G 575, $\mathrm{f}^{\circ} 40 \mathrm{v}^{\circ}$.

94. Arch. dép. de Maine-et-Loire, G $575, \mathrm{f}^{\circ} 35$.

95. Mireille VINCENT-CASSY note que " si les prisonniers étaient mis aux fers (ceps), attachés donc, c'est parce que les fermetures des prisons étaient peu sûres, que les évasions étaient fréquentes, et que le geôlier en était tenu pour responsable et risquait la peine qu'aurait subi l'évadé "; "Prison et châtiments à la fin du Moyen Âge ", les marginaux et les exclus..., op. cit., p. 263. 
de placer sous séquestre, surtout les animaux, chez des personnes privées (dont le choix et la rémunération ne sont pas connus); ainsi,

" Jehan Duboys le jeune, Jehan Laye sur ce que l'en disoit contre eulx que violentement par force et en contempuant et mesprisant justice environ le moys de may à ung certain jour ilz allerent querir en la maison de Jehan Cailleteau ung jument qui y avoit esté mise par le sergent en prinson pour ce qu'elle avoit esté trouvée endommaigeant en certain blez laquelle ilz emmenerent oultre le gré et voulanté dudit Cailleteau et coupperent le collier avecques lequel elle estoit atachée ${ }^{96}$ ".

Ce système permet de faire l'économie de l'entretien d'un bâtiment spécialement destiné à recevoir le bétail divagant. A contrario, ce mode d'enfermement paraît difficilement praticable dans le cas d'individus; par ailleurs, nos sources n'en portent aucune trace.

Simple éclairage d'un sujet qu'il reste encore à approfondir et à compléter, nous souhaitons que ces quelques pages aient réussi à lever certaines zones d'ombre. Ainsi, à travers les prisons et les prisonniers, nous découvrons des justices seigneuriales actives et organisées. Elles ne semblent pas pratiquer l'emprisonnement à tout va et respectent, par exemple, les procédures d'appel intentées par les détenus. Si la torture, via la mise à la question extraordinaire, peut entacher un peu le tableau, il n'en demeure pas moins que tous ces agissements sont légaux, strictement réglementés dans les textes comme dans les faits.

\section{Annexe 1 -Sources de la localisation des prisons seigneuriales repérées en Anjou}

Angers $^{97}$ (Maine-et-Loire, ville, cant. d'Angers, arr. d'Angers) : 1Hs B87, registre d'assises de l'Hôpital Saint-Jean, (1463-1474); 1Hs B176, registre d'assises de l'Hôpital Saint-Jean (1380-1391); H22, registre d'assises de Saint-Aubin, (1399-1407).

Avrillé (Maine-et-Loire, commune, cant. d'Angers, arr. d'Angers) : G891, registre d'assises prieuré de la Haie-aux-Bonhommes, (1358-1380).

Bauné (Maine-et-Loire, cne, cant. de Seiches-sur-Loir, arr. d'Angers) : 34J91, registre d'assises de Briançon, (1474-1514).

Brain-sur-Longuenée (Maine-et-Loire, commune, cant. du Lion-d'Angers, arr. de Segré) : 16J1, (1403-1523).

Briollay (Maine-et-Loire, commune, cant. de Tiercé, arr. d'Angers) : $1^{\mathrm{E}} 1174$, registre d'assises de Sautré ${ }^{98},(1464-1506)$.

96. Arch. dép. de Maine-et-Loire, 1Hs B 87, f 77 (1466).

97. Ce ne sont pas les seules prisons existant à Angers. Ainsi, on pourrait y ajouter la prison du chapitre Saint-Laud où le 19 août 1488, un sous-chantre est incarcéré pour avoir injurié le chantre (Arch. dép. de Maine-et-Loire, G 913, f ${ }^{\circ} 211$ ).

98. Dans un même registre nous avons trouvé l'existence d'une prison ailleurs que dans la seigneurie dont il est question. Ainsi, au $\mathrm{f}^{\circ} 42$ nous pouvons lire " interrogé pourquoy il s'en alla des prisons de Veriné dit que ce fut pour ce que Michau Levenier procureur de la court luy devoit chose et aussi qu'il tira huis de ladite prison ouverte et autre chose non deposé ». Vérigné est un fief dépendant de Briollay. 
Cheffes-sur-Sarthe (Maine-et-Loire, commune, cant. de Tiercé, arr. d'Angers) : H555, (1495-1525).

Chemiré-sur-Sarthe (Maine-et-Loire, commune, cant. de Châteauneuf-sur-Sarthe, arr. de Segré) : G157 ${ }^{99}$, registre d'assises de Morannes (1463-1524).

Cheviré-le-Rouge (Maine-et-Loire, commune, cant. de Baugé, arr. de Baugé) : 8J62, (1450-1489); 8J63, (1475-1487).

Corzé (Maine-et-Loire, commune, cant. de Seiches-sur-Loir, arr. d'Angers) : 8J95, (1472-1476).

Cunault (Maine-et-Loire, bourg, commune de Trèves-Cunault, cant. de Gennes, arr. de Saumur) : 15G19, (1450-1528).

Daumeray (Maine-et-Loire, commune, cant. de Durtal, arr. de d'Angers) : G1999, (1465-1546); G575, registre d'assises de Saint-Denis d'Anjou, $\mathrm{f}^{\circ} 243 \mathrm{v}^{\circ}$.

Feneu (Maine-et-Loire, commune, cant. de Tiercé, arr. d'Angers) : $1^{\mathrm{E}} 1174$, registre d'assises de Sautré, (1464-1506); G443, registre d'assises du Coudray (1430-1509).

Huillé (Maine-et-Loire, commune, cant. de Durtal, arr. d'Angers) : H1056, (14561466); G575, registre d'assises de Saint-Denis d'Anjou, $\mathrm{f}^{\circ} 243$.

Jarzé (Maine-et-Loire, commune, cant. de Seiches-sur-Loir, arr. d'Angers) : ${ }^{\mathrm{E}} 818$, registre d'assises de Montplacé, (1481-1512); 8J14, (1480-1500).

Louerre (Maine-et-Loire, commune, cant. de Gennes, arr. de Saumur) : G302, registre d'assises de Bourgalesme, (1470-1507).

Montguillon (Maine-et-Loire, commune, cant. de Segré, arr. de Segré) : 190J5, registre d'assises de la Bourgonnière, (1478-1541).

Morannes $^{100}$ (Maine-et-Loire, commune, cant. de Durtal, arr. d'Angers) : G152 (14461465), G157 (1463-1524).

Ponts-de-Cé (Maine-et-Loire, commune, cant. d'Angers, arr. d'Angers) : H83, (1482).

Saint-Denis-d'Anjou (Mayenne, commune, cant. de Bierné, arr. de ChâteauGontier) : G575, (1501-1511); G576 (1513).

99. Au $\mathrm{f}^{\circ} 99$, on peut lire « Jehan Bellier Vs pour finance de procès et s'en aller sans jour sur ce que l'on disoit contre luy qu'il avoit brisé les prinsons de Beaumont et en enmenent ses bestes ". D'après, Célestin PORT, Dictionnaire historique, topographique et biographique de Maine-et-Loire, 4 tomes, Paris, Angers, 1874-1878, rééd., Angers, 1965, p. 260, Beaumont est un fief appartenant à la commune de Chemiré-sur-Sarthe. Il s'agit de l'une des quatre baronnies de la Crosse, relevant de l'évêché d'Angers. Le nom primitif était Gratecuisse, elle appartenait à la famille de Craon; Marie de Craon, dame de Chasselay l'apporta en mariage à Robert de Brienne, vicomte de Beaumont et la baronnie prit le nom de Beaumont. Gilles de Retz la vendit en 1433 à Hardouin de Bueil évêque d'Angers qui la légua à son évêché.

100. Dans ce registre, $f^{\circ} 43 v^{\circ}$, on apprend l'existence d'une prison située à Précigné (Sarthe, cne, cant. de Sablé, arr. La Flèche, voir la carte) : " Guillaume Fautrart IIs VId pour finance de procès sur ce que l'on disoit contre lui qu'il avoit prins bestes en la chastelenie deciens et icelles menées en prinson à Précigné et a renoncé avoir droit du faire. " 
Prisons et prisonniers en Anjou au bas Moyen Âge

Annexe 2 - Parcours criminel des délinquants

\begin{tabular}{|l|l|}
\hline $\begin{array}{l}\text { cote } \\
\text { nom-prénom } \\
\text { antécédents }\end{array}$ & JE1174 \\
délits & Jean Chopin \\
$\begin{array}{l}\text { date de l'information } \\
\text { évasion } \\
\text { question extraordinaire } \\
\text { appel }\end{array}$ & Chasse sans droits, vols de gibiers. \\
\hline
\end{tabular}

\begin{tabular}{|l|l|}
\hline $\begin{array}{l}\text { cote } \\
\text { nom - prénom } \\
\text { antécédents } \\
\text { délits } \\
\text { date de l'information } \\
\begin{array}{l}\text { évasion } \\
\text { question extraordinaire } \\
\text { appel }\end{array}\end{array}$ & Gillet Veillon \\
\hline
\end{tabular}

\begin{tabular}{|c|c|}
\hline $\begin{array}{l}\text { cote } \\
\text { nom - prénom } \\
\text { antécédents } \\
\text { délits } \\
\text { date de l'information } \\
\text { évasion } \\
\text { question extraordinaire } \\
\text { appel }\end{array}$ & $\begin{array}{l}\text { H83 } \\
\text { Jean Bouget } \\
\text { Il y a } 7 \text { ans peine corporelle pour le vol d'une jument. } \\
\text { De nombreux vols : animaux, linges, céréales, vin, linges... et diverses } \\
\text { arnaques sur la vente de marchandises. } \\
\text { Information du } 16 \text { octobre } 1482 \text { au } 18 \text { novembre } 1482 . \\
\text { S'échappe une fois de prison, repris, il est mis " es prisons de la } \\
\text { Mairie " d'Angers puis " en la chartre de monseigneur le sénéchal } \\
\text { d'Aniou " puis retour à la prison de Saint-Denis. } \\
\text { A été mis à la question le } 11 \text { novembre } 1482 \text {. }\end{array}$ \\
\hline
\end{tabular}

\begin{tabular}{|l|l|}
\hline $\begin{array}{l}\text { cote } \\
\text { nom - prénom } \\
\text { antécédents } \\
\text { délits }\end{array}$ & $\begin{array}{l}\text { G575 } \\
\text { Grégoire le Taillandier }\end{array}$ \\
& $\begin{array}{l}\text { Crime d'empoisonnement contre Jeannine sa première femme et René } \\
\text { Geslin mari de sa seconde femme Guillemine la Robelotte, plus divers } \\
\text { vols (animaux, vin...). } \\
\text { Information du 14 mai } 1501 \text { au } 3 \text { décembre } 1501 . \\
\begin{array}{l}\text { date de l'information } \\
\text { évasion } \\
\text { question extraordinaire } \\
\text { appel }\end{array}\end{array}$ \\
\hline
\end{tabular}

\begin{tabular}{|l|l|}
\hline $\begin{array}{l}\text { cote } \\
\text { nom-prénom } \\
\text { antécédents } \\
\text { délits }\end{array}$ & $\begin{array}{l}\text { G575 } \\
\text { Guillemine la Robelotte }\end{array}$ \\
$\begin{array}{l}\text { date de l'information } \\
\text { évasion } \\
\text { question extraordinaire d'empoissonnement contre son mari René et contre Jeannine } \\
\text { femme de Grégoire le Taillandier. } \\
\text { appel }\end{array}$ & $\begin{array}{l}\text { Information du 11 août } 1501 \text { au } 13 \text { juin } 1502 . \\
\text { Confesse une évasion grâce à l'aide de Michau Trochon. } \\
\text { A été mise à la question le 14 décembre 1501, le 15 mars } 1502 \text { à Angers } \\
\text { et le 4 juin 1502 à Paris. } \\
\text { A fait appel d'un premier jugement, elle est transférée aux prisons du } \\
\text { palais d'Angers puis de là rejoint les prisons du palais à Paris. }\end{array}$ \\
\hline
\end{tabular}




\begin{tabular}{|l|l|}
\hline $\begin{array}{l}\text { cote } \\
\text { nom-prénom } \\
\text { antécédents } \\
\text { délits } \\
\text { vols } \\
\text { date de l'information } \\
\text { évasion } \\
\text { question extraordinaire } \\
\text { appel }\end{array}$ & G575 \\
Jacquet le Corvaisier \\
Complice des empoisonnements de René et Jeannine plus divers. \\
Information du 3 décembre 1501 au 14 décembre 1501. \\
\hline
\end{tabular}

\begin{tabular}{|l|l|}
\hline $\begin{array}{l}\text { cote } \\
\text { nom-prénom } \\
\text { antécédents } \\
\text { délits } \\
\text { date de l'information } \\
\text { évasion } \\
\text { question extraordinaire } \\
\text { appel }\end{array}$ & $\begin{array}{l}\text { G575 } \\
\text { Michau Jouenneaux }\end{array}$ \\
& Juste une date : 23 décembre 1503. \\
& $\begin{array}{l}\text { A fait appel doit être conduit en la cour du parlement de Paris et } \\
\text { ramené le cas échéant à Saint-Denis. }\end{array}$ \\
\hline
\end{tabular}

\begin{tabular}{|l|l|}
\hline $\begin{array}{l}\text { cote } \\
\text { nom-prénom } \\
\text { antécédents }\end{array}$ & G575 \\
& $\begin{array}{l}\text { Jean Brulle } \\
\text { En prison une fois pour coups et blessure contre sa femme, est sorti en } \\
\text { payant l'amende. } \\
\text { délits } \\
\begin{array}{l}\text { date de l'information vols : argent, linges... } \\
\text { évasion } \\
\text { question extraordinaire } \\
\text { appel }\end{array}\end{array}$ \\
\hline
\end{tabular}

\begin{tabular}{|l|l|}
\hline $\begin{array}{l}\text { cote } \\
\text { nom-prénom } \\
\text { antécédents } \\
\text { délits } \\
\text { date de l'information } \\
\text { évasion } \\
\text { question extraordinaire } \\
\text { appel }\end{array}$ & Mathurin Gruau \\
\hline
\end{tabular}

\begin{tabular}{|l|l|}
\hline $\begin{array}{l}\text { cote } \\
\text { nom-prénom } \\
\text { antécédents }\end{array}$ & G575 \\
& $\begin{array}{l}\text { André Pineau } \\
\text { Il y a } 5 \text { ou } 6 \text { ans emprisonné à Château-Gontier pour bagarre, sort en } \\
\text { acquittant l'amende. }\end{array}$ \\
$\begin{array}{l}\text { délits } \\
\text { date de l'information } \\
\text { évasion } \\
\text { question extraordinaire } \\
\text { appel }\end{array}$ & \begin{tabular}{l} 
Information du 13 août 1511 au 23 août 1511. \\
\hline
\end{tabular} \\
\hline
\end{tabular}

\begin{tabular}{|l|l|}
\hline $\begin{array}{l}\text { cote } \\
\text { nom-prénom } \\
\text { antécédents }\end{array}$ & G575 \\
Jean Pelart \\
$\begin{array}{l}\text { délits y a } 2 \text { ans emprisonné à Saint-Denis pour avoir volé du blé qui était la } \\
\text { dîme de la cure de Saint-Denis. } \\
\begin{array}{l}\text { Divers vols. } \\
\text { évasion } \\
\text { question extraordinaire } \\
\text { appel }\end{array}\end{array}$ \\
\hline
\end{tabular}


Prisons et prisonniers en Anjou au bas Moyen Âge

\begin{tabular}{|l|l|}
\hline $\begin{array}{l}\text { cote } \\
\text { nom-prénom } \\
\text { antécédents }\end{array}$ & $\begin{array}{l}\text { G575 } \\
\text { Michel Priet } \\
\text { Il y a } 6 \text { ans emprisonné } 3 \text { jours à Huillé pour avoir volé la vendange du } \\
\text { châtelain, sort en payant un boisseau de froment; a aussi fait } 3 \text { jours de } \\
\text { prison à Daumeray pour bagarre. } \\
\text { Divers vols : jument, chevaux, vaisselle, draps de laine. } \\
\text { Information du } 20 \text { janvier } 1511 \text { au } 28 \text { janvier } 1511 .\end{array}$ \\
$\begin{array}{l}\text { délits } \\
\text { date de l'information } \\
\text { évasion } \\
\begin{array}{l}\text { question extraordinaire } \\
\text { appel }\end{array}\end{array}$ & \\
\hline
\end{tabular}

\section{RÉSUMÉ}

À travers un type de source stéréotypé, les registres d'assises, nous découvrons quelques aspects des prisons et des prisonniers en Anjou au bas Moyen Âge. Il s'agit, à travers cette étude, de s'intéresser strictement aux prisons relevant des justices seigneuriales. Par ailleurs, la consultation de la coutume d'Anjou et du Maine a permis d'apporter une dimension supplémentaire aux thèmes abordés. Ce sujet a donc pu être abordé sous deux angles, l'un théorique et l'autre plus pratique.

\section{ABSTRACT}

Through one type of stereotyped data such as justice records we unveil some interesting facts on prisons and prisoners' conditions in late Middle Ages Anjou. The main focus of that research is on prisons run by lords with their own justice system. Also, a closer at Anjou and Maine customs brought an other dimension to the themes of my study. As a result, the subject is treated on both theoretical and pratical aspects. 
